\title{
Influência das variáveis meteorológicas na saúde: análise das internações por pneumonia em crianças
}

Existe uma prevalência nos registros hospitalares por doenças respiratórias, em especial nas crianças. A pneumonia é uma das morbidades respiratórias com maiores registros de morbimortalidade no Brasil e no município em estudo. Objetivou-se com esta pesquisa analisar a influência das variáveis meteorológicas nas internações por pneumonia em crianças. A atual pesquisa se trata de um estudo epidemiológico, descritivo de corte transversal com abordagem quantitativa e descritiva. Para a análise estatística dos dados foi ajustado o modelo binomial negativo pertencente à classe dos modelos lineares generalizados, adotando-se um nível de significância de 5\%, com base na plataforma estatística R. Estimou-se que o número médio de casos de pneumonia aumente aproximadamente 48 \% a cada grau centígrado de aumento da temperatura média do ar e diminua aproximadamente $32 \%$ a cada grau centígrado de aumento da temperatura máxima do ar, diminuindo também em torno de $3 \%$ a cada $1 \%$ de aumento acima da média da umidade relativa, em relação a variável vento espera-se que o número de casos de pneumonia aumente em torno de $36 \%$ com o aumento na velocidade do vento. Avaliar o risco para a saúde da população em razão dos dados climáticos é um passo importante para o planejamento e a implementação de ações. Os gestores da saúde pública podem utilizar o modelo de previsão para preparar os serviços de saúde para receber crianças em períodos em que ocorrem maiores registros de casos de pneumonia.

Palavras-chave: Variáveis climáticas; Doenças respiratórias; Hospitalizações; Crianças.

\section{Influence of meteorological variables in health: analysis of pneumonia innovations in children}

\begin{abstract}
There is a prevalence in hospital records of respiratory diseases, especially in children. Pneumonia is one of the respiratory morbidities with the highest morbidity and mortality rates in Brazil and in the municipality to be studied. The objective of this research was to analyze the influence of meteorological variables upon hospitalizations for pneumonia in children. The current research is an epidemiological, cross-sectional descriptive study with a quantitative and descriptive approach. For the statistical analysis of the data the negative binomial model belonging to the class of generalized linear models was adjusted, adopting a level of significance of $5 \%$, based on the statistical platform R. It was estimated that the average number of pneumonia cases increased approximately $48 \%$ at each degree centigrade increase in mean air temperature and decrease approximately $32 \%$ at each degree centigrade increase in maximum air temperature, also decreasing by about $3 \%$ at every $1 \%$ increase above the relative humidity average, as to the variable wind the number of pneumonia cases is expected to increase by around $36 \%$ with the increase of wind speed. Assessing the health risk of the population due to climate data is an important step in planning and implementing actions. Public health managers can use the predictive model to prepare health services to receive children in periods when there are major records of pneumonia cases.
\end{abstract}

Keywords: Climate Variables; Respiratory diseases; Hospitalizations; Children.

Topic: Epidemiologia e Saúde Ambiental

Reviewed anonymously in the process of blind peer
Received: $12 / 04 / 2019$

Approved: 27/05/2019
Juliana Meira de Vasconcelos Xavier (iD)

Universidade Federal de Campina Grande, Brasil

http://lattes.cnpq.br/2221607460684235

http://orcid.org/0000-0003-0603-1943

juliana-mvasconcelos@hotmail.com

Patricio Marques de Souza (D)

Universidade Federal de Campina Grande, Brasil

http://lattes.cnpq.br/0395671025242338

http://orcid.org/0000-0002-2064-3818

patriciomsouza@gmail.com

Fabrício Daniel dos Santos Silva (id

Universidade Federal de Alagoas, Brasil

http://lattes.cnpq.br/9324908747974694

http://orcid.org/0000-0002-3185-6413

fabricio.santos@icat.ufal.br

\author{
Ricardo Alves de Olinda (iD \\ Universidade Estadual da Paraíba, Brasil \\ http://lattes.cnpq.br/7767223263366578 \\ http://orcid.org/0000-0002-0509-8428 \\ ricardo.estat@yahoo.com.br \\ Luana Andrade Lima Querino (ic) \\ Universidade Federal de Campina Grande, Brasi \\ http://lattes.cnpq.br/3979403454435718 \\ http://orcid.org/0000-0001-5874-6006 \\ luanaandradelima@gmail.com
}

Referencing this:

XAVIER, J. M. V.; SOUZA, P. M.; SILVA, F. D. S.; OLINDA, R. A.; QUERINO, L. A. L.. Influência das variáveis meteorológicas na saúde: análise das internações por pneumonia em crianças. Revista lbero Americana de Ciências Ambientais, v.10, n.3, p.30-41, 2019. DOI: http://doi.org/10.6008/CBPC2179-6858.2019.003.0004 


\section{INTRODUÇÃO}

Há uma preocupação crescente em compreender o impacto das variáveis meteorológicas na epidemiologia das doenças. Os fatores ambientais atuam sobre a dinâmica da ocorrência de várias morbidades em especial das doenças respiratórias, aumentando as taxas de morbidade e intensificação da doença. Dentre os fatores ambientais destaca-se as variáveis meteorológicas, tais como temperatura, umidade relativa do ar, precipitação pluviométrica, pressão atmosférica e velocidade dos ventos.

Diversos estudos evidenciaram efeitos de fatores climáticos na ocorrência de doenças cerebrovasculares, cardiovasculares, respiratórias, reumáticas e infecciosas transmitidas por vetores, aumento de casos de meningite virais (CURRIERO et al., 2001; FAÇANHA, 2005; GONZÁLEZ et al., 2008; LIMA et al., 2008; MENDONÇA et al., 2008; LIN et al., 2009; MIRANDA et al., 2007; SILVA JÚNIOR et al., 2011). As variações climáticas podem contribuir para o desencadeamento e agravamento de diversas outras enfermidades quando somados a outros fatores de risco ou condições pré-existentes (MURARA et al., 2010; PITTON et al., 2004).

O tempo meteorológico é algo que varia muito sobre a face da Terra, sendo o estado instantâneo da atmosfera ou a soma total das condições atmosféricas de dado local, em determinado tempo cronológico. $O$ clima é uma generalização ou a integração das condições do tempo para certo período, em determinada área, envolvendo, portanto, um maior número de dados do que as condições médias de tempo em uma determinada área (VIANELLO et al., 2012). Como componente da natureza o clima também é considerado um recurso natural, sendo um do mais importante recurso à disposição do homem (AYOADE, 2012; CONTI, 2007).

Pesquisas que relacionam clima e saúde e/ou clima e bem-estar estão incluídas no campo da Bioclimatologia Humana. Conforme Pitton et al. (2004), "bioclimatologia é a ciência que se dedica ao estudo de influências do ambiente atmosférico ao homem. Estas influências podem ser termais, barométricas, hídricas, e também as causadas pela composição do ar ambiente". Sendo importante destacar que tais influências acarretam em surgimento de enfermidades e mudanças comportamentais no ser humano.

Pequenas variações climáticas são em geral bem toleradas. Entretanto situações extremas de aumento ou diminuição de temperatura estão relacionadas a ocorrência de doenças de diversas etiologias, em especial as doenças respiratórias. Tais efeitos podem ocorrer em pessoas predispostas, tais como idosos, crianças e portadores de doenças crônicas (CONTI, 2007; PITTON et al., 2004).

Em todo o mundo as doenças respiratórias agudas ou crônicas são apontadas como uma das principais causas de morbimortalidade no homem, acometendo principalmente crianças e idosos. Dentre elas se destacam a asma, a pneumonia e a bronquite, doenças respiratórias que afetam as vias aéreas inferiores, configurando um problema mundial de saúde (DATASUS, 2018).

Diante do exposto percebe-se que as alterações das variáveis meteorológicas concorrem de forma efetiva para ocorrência de doenças respiratórias no ser humano, em especial nas crianças. Diversos fatores podem propiciar a ocorrência de doenças respiratórias em crianças, dentre eles as variações climáticas. 
No Brasil as doenças respiratórias agudas e crônicas ocupam posição de destaque. Esse grupo de doenças apresenta-se entre as principais causas de internações no Sistema Único de Saúde - SUS. Os dados de morbidade hospitalar no Brasil são compilados no Sistema de Informação Hospitalar (SIH). Este sistema nacional, como o nome sugere, armazena informações de todas as internações hospitalares que acontecem no SUS.

Segundo Hetzel et al. (2008), as infecções respiratórias virais podem ocorrer em qualquer época do ano, entretanto apresentam maior incidência no inverno do que no verão. Essas acometem pessoas de qualquer idade, entretanto são mais incidentes entre crianças do que em adultos. A vulnerabilidade de crianças em relação a outros grupos etários pode ser explicada pela imaturidade do sistema imunológico associada ao menor calibre das vias aéreas que dificulta a remoção de partículas estranhas nas vias respiratórias (MACEDO et al., 2007; O' BRODOVICH et al., 1998).

Objetivou-se com esta pesquisa analisar a influência das variáveis meteorológicas em internações hospitalares de crianças menores de 9 anos acometidas por doenças do trato respiratório inferior (pneumonia, asma e bronquite/bronqueolite) em Campina Grande, localizada no estado da Paraíba, além de quantificar os casos de internações hospitalares.

\section{MATERIAIS E MÉTODOS}

Área e período do estudo - Esta pesquisa foi realizada com dados relativos ao município de Campina Grande, localizado no Estado da Paraíba (PB), do período de janeiro de 1998 a dezembro de 2017, equivalente há vinte anos. O município de Campina Grande está a uma altitude média de 555 metros acima do nível do mar, com 7 $13^{\prime} 11^{\prime \prime}$ de latitude Sul e $35^{\circ} 52^{\prime} 31^{\prime \prime}$ de longitude Oeste, distante a $120 \mathrm{~km}$ da Capital do Estado, João Pessoa. A área do município de Campina Grande abrange 593,026 Km², contando com uma população estimada em 407.754 habitantes (densidade demográfica de 648,31 hab./ $/ \mathrm{km}^{2}$ ), segundo dados do Instituto Brasileiro de Geografia e Estatística (IBGE, 2018). Está inserido na unidade geoambiental do Planalto da Borborema e incluído na área geográfica de abrangência do semiárido brasileiro. Apesar disso, por estar acima de quinhentos metros de altitude, possui um clima com temperaturas mais moderadas, considerado Tropical com estação seca (As, de acordo com a classificação climática de Köppen-Geiger). A estação chuvosa se inicia em maio com término em setembro, podendo se estender até outubro

Os serviços de saúde municipal contam com 728 estabelecimentos sendo 217 públicos, com 90 Unidades Básicas de Saúde da Família. Conta com 1.593 leitos para internações sendo 1.231 credenciados pelo Sistema Único de Saúde - SUS. A atual pesquisa utilizou um estudo transversal de abordagem quantitativa e descritiva. Os dados foram de fonte secundária, relativos às séries de dados meteorológicos obtidas no Instituto Nacional de Meteorologia (INMET) e do número de hospitalizações por pneumonia do período de tempo estudo: janeiro de 1998 a dezembro de 2017, 240 meses em 20 anos, os dados epidemiológicos foram coletados no site do DATASUS, sendo necessária a tabulação e sistematização dos mesmos, com o auxílio do Excel ou outra ferramenta de conversão, como o software TAB Win 32. 
Os números de casos de hospitalizações de crianças menores de nove anos em Campina Grande (PB), foram do banco de dados do Departamento de Informação do Sistema Único de Saúde (DATASUS), Sistemas de Informação em Saúde (SIS) através do Sistema de Informações Hospitalares (SIH). A pesquisa utilizou dados administrativos obtidos de documentação oficial de domínio público. Estes dados foram utilizados exclusivamente para objetivo desta pesquisa e as informações preparadas e apresentadas de maneira coletiva de tal forma que nenhum resultado se referiu a um indivíduo nominal, tampouco implicaram quaisquer prejuízos para as pessoas ou instituições envolvidas. Por não se tratar de pesquisa direta com seres humanos foi dispensado o encaminhamento ao Comitê de Ética e Pesquisa Científica (CEP).

\section{Análise Estatística dos Dados}

Um desafio existente na avaliação da influência das variáveis climáticas nos casos de internações hospitalares é encontrar um modelo estatístico capaz de considerar todos os fatores envolvidos. Sendo assim, para quantificar os efeitos que as variáveis meteorológicas (temperatura, umidade relativa do ar, precipitação pluviométrica, pressão atmosférica, velocidade dos ventos e evaporação) exercem no número de internações hospitalares, foram testados dois modelos pertencentes à classe dos Modelos Lineares Generalizados (MLGs), os modelos de regressão Poisson e Binomial Negativo, adotando-se um nível de significância de $5 \%(p<0,05)$ (SANTOS, 2015).

Os dados meteorológicos foram organizados em planilhas do software Excel, juntamente com os registros de internações hospitalares por pneumonia em crianças de 0 a 9 anos, fornecidos pelo Departamento de informática do SUS - DATASUS e trabalhados pelo software estatístico R (R CORE TEAM, 2018), os quais foram tratados e analisados.

A análise descritiva dos dados em termos de porcentagens da variável dependente (casos de internação hospitalar por pneumonia) e independentes (variáveis meteorológicas) e foi obtida pela medida de tendência central (média, mediana), dispersão (variância e desvio padrão), de forma (assimetria e curtose) e o coeficiente de variação (CV);

Em seguida, por se tratar de dados de contagem, após o ajuste do modelo de regressão de Poisson, foi necessário ajustar o modelo de regressão binomial negativa, mais adequado para tratar dados com variância superior à média condicional, foi elaborado com a adição de um novo parâmetro que reflete a heterogeneidade não observada.

Para avaliar o ajuste do modelo, realizou-se uma análise dos resíduos através dos gráficos normais de probabilidade juntamente com o teste de normalidade Anderson-Darling para todas as variáveis para verificar a adequabilidade do ajuste do modelo aos dados. Por fim, as análises foram realizadas com o auxílio da plataforma estatística R (R CORE TEAM, 2014).

\section{RESULTADOS E DISCUSSÃO}

Nesta pesquisa foi possível analisar a relação entre os casos de internações por pneumonia em crianças menores de nove anos no município de Campina Grande (PB) e as variáveis climáticas. Desta forma, 
o propósito básico deste estudo foi determinar possíveis relações entre elementos climáticos, tais como: temperatura do ar, umidade relativa do ar e precipitação pluviométrica, velocidade do vento, pressão atmosférica, evaporação e a ocorrência da pneumonia, com base em uma avaliação das correlações existentes.

Os casos de pneumonia no município de Campina Grande/PB, relativos aos dados do Sistema de Internação Hospitalar do Sistema Único de Saúde (SIH/SUS), foram distribuídos de acordo com cada mês nos últimos vinte anos. No período de vinte anos da série em estudo (janeiro de 1998 a dezembro de 2017), foram registrados um total de 22.203 internações hospitalares por pneumonia. Ao avaliar essas internações por faixa etária se observou 6.572 internações na faixa etária de 0 a 12 meses (29,5\%); 11.058 internações na faixa etária de 1 a 4 anos (49,8\%); 3.443 internações na faixa etária de 5 a 9 anos (15,5\%).

Observa-se que os anos que apresentaram maiores registros de internações hospitalares, para os casos de pneumonia foram os anos de 1998 e 1999, com 2.029 e 1.953 registros respectivamente, quanto a faixa etária das crianças que apresentaram- se mais suscetível as internações estão aquelas entre 1 a 4 anos. Em relação aos meses com maior número de registro foram: maio (2487) e junho (2485). O ano que apresentou o menor número de registro hospitalar foi o ano de 2012 com 523 casos.

Analisando-se as taxas de internações por pneumonia para o município de Campina Grande, tanto mensal quanto sazonalmente, há um comportamento que se repete nos anos avaliados, onde as menores taxas de internações são percebidas nos meses de verão, após o qual se elevam durante o outono, e inverno, decaindo novamente durante a primavera.

Alguns fatores podem contribuir para que o frio aumente a incidência de infecções no trato respiratório, dentre eles, o favorecimento da transmissibilidade de vários vírus durante o registro de baixas temperaturas, diminuição da imunidade do organismo, favorecendo a ocorrência de infecções respiratórias (FALANGAS et al., 2008).

Segundo Hetzel et al. (2008), as infecções respiratórias virais podem ocorrer em qualquer época do ano, entretanto apresentam maior incidência no inverno do que no verão. Essas acometem pessoas de qualquer idade, entretanto são mais incidentes entre crianças do que em adultos. A vulnerabilidade de crianças em relação a outros grupos etários pode ser explicada pela imaturidade do sistema imunológico associada ao menor calibre das vias aéreas que dificulta a remoção de partículas estranhas nas vias respiratórias, favorecendo a evolução do quadro para formas graves de pneumonia, com disfunção respiratória significativa e consequente necessidade de hospitalização (MACEDO et al., 2007; O' BRODOVICH et al., 1998).

Em relação a variável climática temperatura, as temperaturas, média máxima e mínima apresentaram oscilações nas médias mensais e anuais no município de Campina Grande (PB) a temperatura média oscilou de $21,6^{\circ} \mathrm{C}$ no mês de julho a $24,9^{\circ} \mathrm{C}$ no mês de março dos anos de 1998 a 2017.0 ano de 2004 apresentou a menor temperatura média $\left(23,09^{\circ} \mathrm{C}\right)$ e 1998 a maior temperatura média $\left(24,31^{\circ} \mathrm{C}\right)$. Em relação a temperatura máxima houve uma oscilação de $\left(25,6^{\circ} \mathrm{C}\right)$ no mês de julho e no mês de dezembro $\left(30,7^{\circ} \mathrm{C}\right)$, $\mathrm{O}$ ano de 1998 apresentou a maior registro de T máx. $\left(30,02^{\circ} \mathrm{C}\right)$. No que se refere a temperatura mínima, essa 
oscilou de $18,5^{\circ} \mathrm{C}$ no mês de agosto a $21,5^{\circ} \mathrm{C}$ em março, o ano de $2004\left(19,68^{\circ} \mathrm{C}\right)$ apresentou menor registro de Temperatura mínima.

Para Omonijo et al. (2011), a temperatura máxima do ar está estatisticamente associada a pneumonia, sinusite e a asma, já a umidade relativa do ar é responsável pelo grande número de asma e amigdalite. A média da umidade relativa do ar variou de $71,9 \%$ em novembro a $83,8 \%$ em junho, no município de Campina Grande (PB). O ano de 1999 apresentou a menor média anual (71,50\%) e o ano de 2011 (82,93\%) a maior média.

O vento no município de Campina Grande (PB) variou de 3,2 m/s/mês nos meses de maio, junho e julho a 4,3 m/s /mês novembro. Os anos de 2009 (3,38 m/s /ano) e 1998 (4,32 m/s/ano) apresentaram respectivamente, a menor e maior valores de velocidade de vento. Em Campina Grande (PB), como os dados são de contagem pôde-se pensar inicialmente num modelo de Poisson. Em que Pneumoniai denota o número de internações hospitalares por pneumonia, tal que Pneumoniai $\sim \mathrm{P}(\mu i)$ em que:

$$
\begin{aligned}
& \log \mu i=60+61 \text { tempmed } i+62 \text { tempmáxi }+ \text { b3tempmini+ b4precipi }+ \text { b5umidi+ b6pressaoi+ } \\
& \text { B7insolai+ b8ventoi+ Bgevaporaçãoi }
\end{aligned}
$$

Para $i=1,2, \ldots, 240$. Entretanto, o ajuste do modelo forneceu $D(y ; \mu)=3.504,40$ para 231 graus de liberdade, indicando fortes indícios de sobredispersão e há evidência significativa de que o ajuste não seja adequado ( $p$-valor $<0,001$ ), o que é confirmado pelo gráfico normal de probabilidade da Figura 1.

Tem-se então um modelo binomial negativo com ênfase na relação entre o número de hospitalizações de ocorrência de pneumonia e as variáveis climáticas em estudo. Em que Pneumoniai BN $(\mu i, \phi)$. O gráfico normal de probabilidades bem como o desvio $D(y ; \mu)=246,48$ fornecem indícios de ajustes adequados ( $p$-valor $=0,2905$ ), com estimativa do parâmetro de dispersão $(\phi)$ igual a 6,14 , ratificando assim, um bom ajuste do modelo aos dados em questão.

Além disso, a distribuição de Poisson assume que eventos ocorrem de maneira independente ao longo do tempo, isto é, que a probabilidade da criança ser consultada e diagnosticada com uma doença respiratória, como por exemplo a pneumonia em estudo pela j-ésima vez é independente do (j+1) -ésimo e (j-1) ésimo diagnóstico. No âmbito da saúde, essa hipótese não apresenta muito sentido, pois uma vez que a criança foi diagnosticada e internada, é provável que não seja realizado um retorno a fim de ser avaliado a eficácia do tratamento.

Além disso, os resultados apresentados na Tabela 1 indicam que as variáveis meteorológicas temperatura média, máxima, umidade relativa do ar e vento foram significativas ao nível de $5 \%$ de probabilidade, no que se refere à explicação da taxa de aumento/decréscimo nos casos de internações hospitalares por pneumonia no período de 1998 a 2017 em Campina Grande (PB).

O coeficiente $\beta_{1}=0,39239$ (Tabela 1 ) indica aumento dos casos de internações por pneumonia em função do aumento da temperatura média, ou seja, existe uma relação positiva entre as variáveis em estudo (Figura 2). Em relação ao coeficiente $\beta 2=-0,3904$, temperatura máxima, observa-se uma relação inversa entre as variáveis em estudo, isso indica que há uma redução dos casos de pneumonia à medida que a 
variável temperatura máxima aumenta. Logo, espera-se que para os meses com maiores registros de temperatura média, haja maiores registros de ocorrência de pneumonia (Figura 3) Sendo assim, exp. $(0,39239)=1,4805$, estima-se que o número médio de casos de pneumonia aumente aproximadamente $48 \%$ a cada grau centígrado de aumento da temperatura média do ar e para temperatura máxima exp. (-0,3904)= 0,6768 estima-se que o número médio de casos de pneumonia diminua aprox. 32\% a cada grau centígrado de aumento da temperatura máxima do ar.
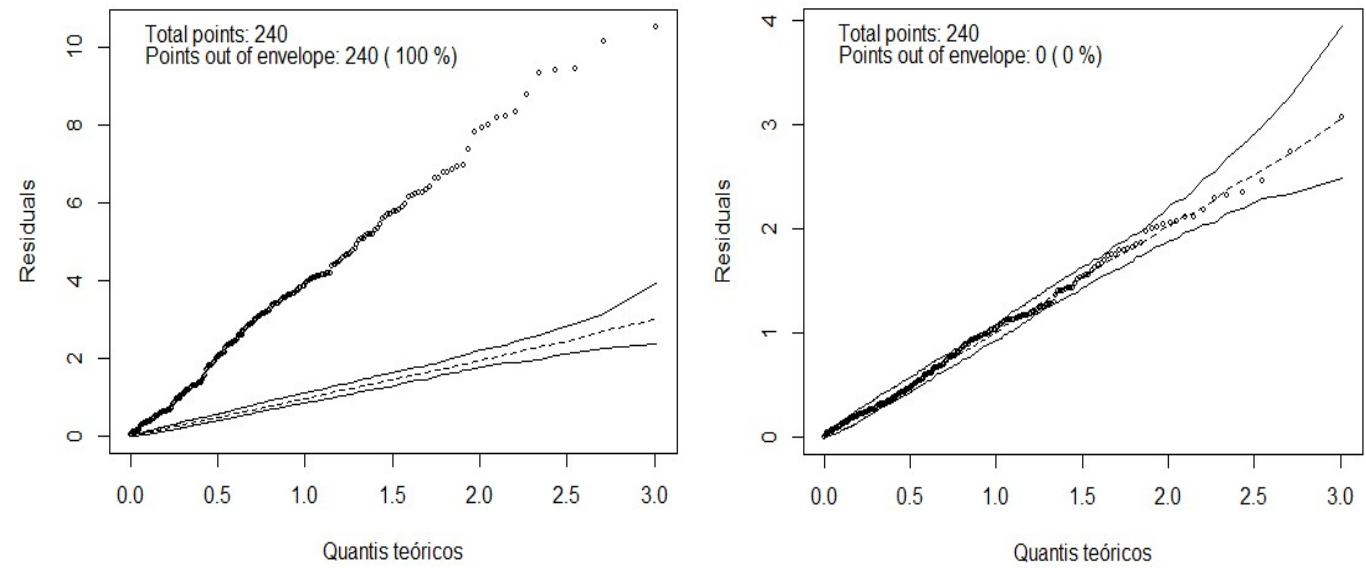

Figura 1: Gráficos normais de probabilidades referentes aos modelos log- linear de Poisson (a) e log-linear binomial negativo (b) ajustados aos dados relacionados as internações hospitalares por pneumonia em crianças menores de 9 anos, no período de 1998 a 2017 em Campina Grande/PB.

Tabela 1: Estimativas dos parâmetros do modelo e seus respectivos erros estandardizados, valor z e o correspondente p-valor para ocorrência de internações hospitalares por pneumonia no período de 1998 a 2017 em Campina Grande (PB).

\begin{tabular}{|l|l|l|l|l|}
\hline Coeficientes & Estimativa & Erro estandardizado & Valor $\mathbf{z}$ & p-valor \\
\hline Intercepto (60) & 7,84106 & 1,57663 & 4,973 & $6,58 \mathrm{e}^{-07 * * *}$ \\
\hline Tmed (61) & 0,39239 & 0,05909 & 6,641 & $3,12 \mathrm{e}^{-11 * * *}$ \\
\hline Tmax (62) & $-0,39040$ & 0,04324 & $-9,029$ & $<2, \mathrm{e}^{-16 * * *}$ \\
\hline UR (63) & $-0,03167$ & 0,01095 & $-2,893$ & $0,00382 * *$ \\
\hline Vento (68) & 0,30796 & 2,988 & $0,00280^{* *}$ \\
\hline
\end{tabular}

Para o município de Ponta Grossa (PR), Pontes et al. (2016) avaliaram os efeitos do clima (através das variáveis climáticas temperatura do ar, precipitação e umidade relativa do ar) nas internações por pneumonia em crianças menores de cinco anos, no período de 1998 a 2013 . O estudo demonstrou forte correlação positiva entre as temperaturas máxima e mínima, evidenciando que essas temperaturas são as que mais influenciam nas internações por pneumonia, ambas altamente significativas. As variáveis umidade relativa do ar e precipitação pluviométrica apresentaram correlação negativa e não mostraram correlação significativa. Diferentemente dos resultados encontrados na atual pesquisa as internações por pneumonia na cidade de Campina Grande, apresentaram correlações estatisticamente significativas para a variável temperatura, porém fracas, além de correlação positiva com a temperatura média e negativa com a temperatura máxima, logo, espera-se que para os meses com maiores registros de temperatura máxima do ar, observam-se os menores índices de hospitalizações por pneumonia na faixa etária em estudo, contrapondo-se aos resultados encontrados para o município de Ponta Grossa. 


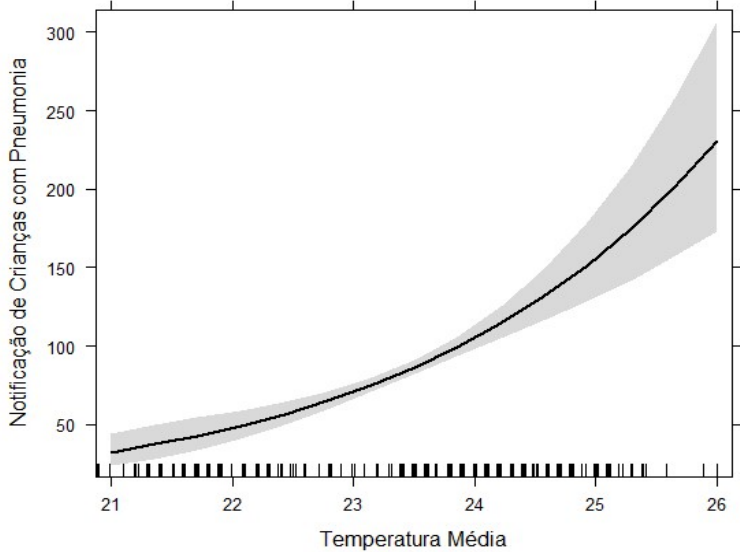

Figura 2: Comportamento dos casos de pneumonia em crianças menores de 9 anos em relação a temperatura média do ar em Campina Grande (PB), 1998 a 2017.

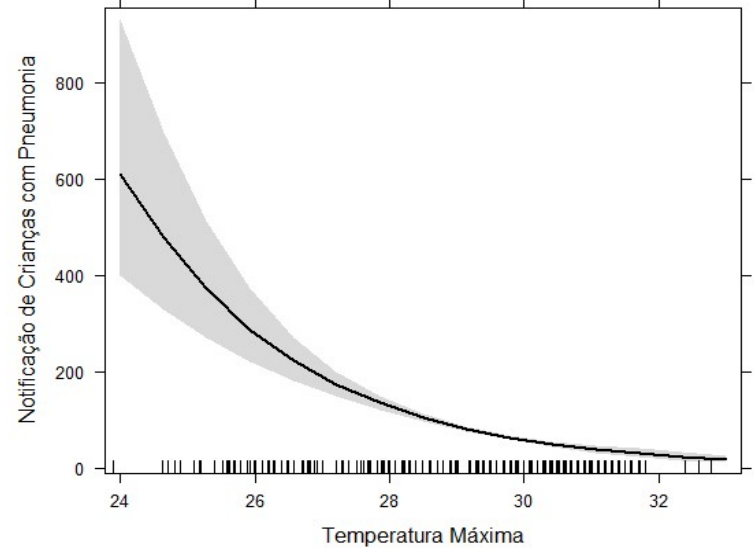

Figura 3: Comportamento dos casos de pneumonia em crianças menores de 9 anos em relação a temperatura máxima do ar em Campina Grande (PB), 1998 a 2017.

Falangas et al. (2008) em pesquisa na Grécia relação à qual correlacionaram variáveis meteorológicas com infecções do trato respiratório (ITR), constataram que após análises estatísticas, houve correlação negativa estatisticamente significante entre a temperatura média semanal e os casos de infecções do trato respiratório, ou seja, os casos de ITR aumentaram à medida que as médias de temperatura diminuíram. Também houve correlação negativa entre o vento e a ocorrência de infecções no trato respiratório superior e inferior. Além disso, houve correlação positiva entre umidade relativa e a ocorrência de infecções do trato respiratório. Contrapondo-se a atual pesquisa que encontrou correlação positiva com a temperatura média, inversa com a umidade relativa do ar e positiva com o vento.

Em Brisbane na Austrália, no período de 2001 a 2010 foi realizado estudo entre a diferença de temperatura máxima e mínima e a diferença média de temperatura de um dia para o outro (a mudança de temperatura) com o número de registro de casos de pneumonia em crianças que evidenciou uma tendência sazonal na pneumonia infantil. Nenhuma associação significativa entre diferença de temperatura máxima e mínima foi observada, entretanto uma queda de temperatura para o dia seguinte foi associada com o aumento de casos de pneumonia, esse efeito durou 3 semanas. $O$ estudo também constatou que havia uma sazonalidade na ocorrência de pneumonia em crianças e o impacto maior no aumento dos registros hospitalares ocorreram no inverno, junho, julho e agosto (XU et al., 2014).

Braga et al. (2002) analisaram o efeito do clima e a mortalidade por doenças respiratórias, em doze cidades dos Estados Unidos. Esses autores perceberam que temperaturas baixas favorecem ao aumento principalmente dos casos de pneumonia. O tempo frio favorece o imunocomprometimento do trato respiratório. Em crianças, por exemplo, a vasoconstrição periférica diminui o movimento ciliar restringindo, assim, a eliminação de patógenos no epitélio. Como as crianças, têm menos capacidade de se adaptar às condições climáticas que mudam repentinamente pode haver um aumentar no risco de adoecimento por doenças respiratórias (TIAN et al., 2017; XU et al., 2012).

Em Hangzhou na China, foi realizado estudo para investigar se a incidência de pneumonia por micoplasma pediátrico (MP) e pelo vírus sincicial respiratório (VSR) estava associada a fatores meteorológicos. Aproximadamente $80 \%$ das infecções por MP ocorreram em crianças com sete anos de 
idade ou menos. No entanto, RSV infectou principalmente bebês, especialmente crianças com seis meses ou menos. A infecção pelo VSR foram predominantemente prevalente no inverno. Tal fato acontece porque bebês e crianças pequenas têm sistemas imunológicos imaturos, que pode ser ainda mais reprimido no inverno. As baixas temperaturas promovem espasmos do trato respiratório e isquemia devido à contração capilar em crianças, resultando em enfraquecimento do movimento ciliar e, consequentemente, enfraquecimento da remoção do VSR no epitélio respiratório (TIAN et al., 2017).

Em Ribeirão Preto (SP), no período de 1978 a 2008, foi analisada a influência das condições climáticas em internações por pneumonia. Para isso, foram coletados dados de temperatura máxima, mínima, precipitação pluvial e umidade relativa do ar. As condições diárias de temperatura mínima com valores inferiores a $15^{\circ} \mathrm{C}$, temperatura média com valores inferiores a $21,5^{\circ} \mathrm{C}$ e amplitude térmica superior a $14,8^{\circ} \mathrm{C}$ associaram-se ao aumento do risco de hospitalizações por pneumonia com significância estatística, A umidade relativa do ar não demonstrou significância estatística (ALEIXO et al., 2014).

O coeficiente relacionado a umidade relativa do $\operatorname{ar} \beta_{3}=-0,03167$, foi negativo, indicando a diminuição dos casos de pneumonia em função do aumento da umidade relativa, ou seja, existe uma relação inversa entre as variáveis em análise. Assim espera-se que, para os meses com maiores registros de umidade relativa do ar, sejam observados menores índices de internações por pneumonia. Isto é, tomando exp. $(-0,03167)=$ 0,9688, estima-se que o número médio de casos por pneumonia diminua em torno de $3 \%$ a cada $1 \%$ de aumento acima da média da umidade relativa, Figura 4.

Em contraposição aos resultados encontrados nessa pesquisa, Andrade et al. (2015) avaliaram que havia maior número de atendimentos às crianças por doenças respiratórias (pneumonia, doenças obstrutivas, Infecções respiratórias agudas e infecções das vias aéreas superiores) quando havia um aumento da umidade relativa do ar, demonstrando uma correlação positiva entre as variáveis em estudo.

Na pesquisa de Botelho et al. (2003) e Alonso et al. (2007) também avaliaram a relação existente entre a umidade relativa e os casos de doenças respiratórias. Observou-se um aumento no adoecimento por doenças respiratórias, incluindo a pneumonia, em períodos com umidade relativa do ar alta, tal fato pode ser explicado por maiores aglomerações de pessoas, proliferação de fungos e ácaros. Tais pesquisas, citadas anteriormente, também diferem dos resultados encontrados no atual estudo que apresentou correlação inversa entre as internações por pneumonia e a umidade relativa do ar.

Em São Paulo (SP) foi realizado um estudo que investigou a associação de internações por pneumonia e gripe com variáveis meteorológicas tais como, temperaturas máximas e mínimas diárias e umidade relativa do ar, o período de estudo foi de 2002 a 2005. Em geral as internações não apresentaram correlação forte, mostrando correlações médias entre julho e setembro de 2004, para a influenza e a pneumonia. Observou-se associação das internações por pneumonia com baixas temperaturas nas madrugadas (47\%) e temperaturas máximas durante a tarde (35\%), nos períodos mais secos do ano. Nesses casos, as internações nesse período são reflexos da umidade do ar abaixo de $30 \%$. Os picos de internação ultrapassam 80 pacientes por dia, após um período anterior de aproximadamente três dias de baixa umidade. Semelhante a achado observado no presente estudo, houve maior proporção de internações por pneumonia 
para os meses com menores registros de umidade relativa do ar. Estes resultados corroboram com os encontrados na atual pesquisa. É importante salientar que, para o bom funcionamento das vias aéreas, há necessidade de certo grau de umidade, que não deverá ser inferior a $60 \%$.

Segundo Lowen et al. (2007), a umidade relativa do ar pode influenciar a ocorrência de doenças respiratórias devido aos seguintes mecanismos: respirar o ar seco pode causar ressecamento da mucosa nasal e torna o hospedeiro mais suscetível a infecções; a exposição prolongada ao ar seco pode contribuir com o crescimento do vírus no trato respiratório superior.

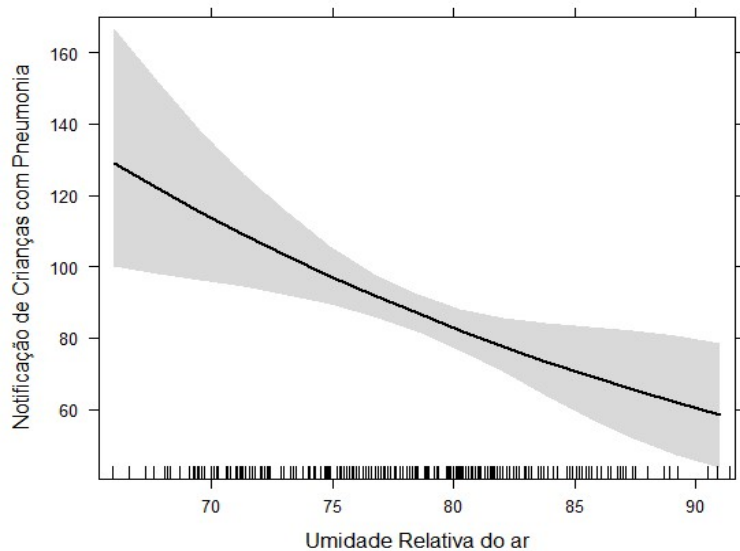

Figura 4: Comportamento dos casos de pneumonia em crianças menores de 9 anos em relação a umidade relativa do ar em Campina Grande (PB), 1998 A 2017.

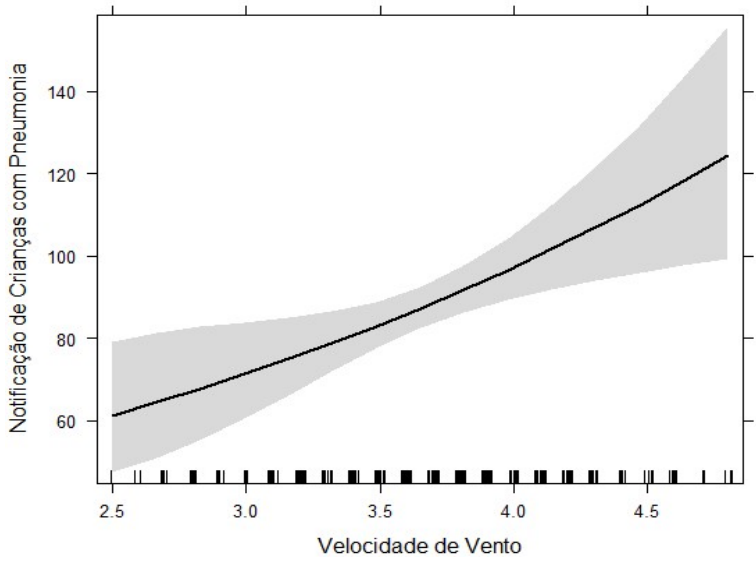

Figura 5: Comportamento dos casos de pneumonia em crianças menores de 9 anos em relação ao vento em Campina Grande (PB), 1998 A 2017.

O coeficiente relacionado ao vento $\beta_{6=0} 0,30796$, indica que há um aumento no registro de hospitalizações por pneumonia à medida que ocorra um aumento na velocidade do vento. Portanto exp. $(0,30796)=1,3606$, estima-se que o número de casos de pneumonia aumente em torno de $36 \%$ a cada aumento na velocidade do vento.

Souza et al. (2012) verificaram em estudo realizado em Campo Grande (MS) no período de 2004 a 2008 o impacto de variáveis climáticas (precipitação, temperatura do ar, umidade e velocidade dos ventos) sobre as internações por pneumonia em lactentes e crianças, mostrou que a diminuição da umidade, precipitação e velocidade dos ventos (altas polares, que se caracterizam por serem frias e secas) favorecem a internações por pneumonia.

$\mathrm{O}$ atual estudo mostrou uma correlação positiva em relação ao vento e o aumento na ocorrência de pneumonia. É conhecida a capacidade do vento em promover dispersão de poluentes e de material particulado, produzindo uma redução de suas concentrações no ar. Provavelmente, os menores valores na velocidade do vento observados no inverno, podem estar associados aos picos de observações de casos registrados da pneumonia, nesse período devido à menor dispersão de agente poluentes e causadores de alergias que podem evoluir para a morbidade em estudo.

\section{CONCLUSÕES}

As variáveis meteorológicas temperatura média, máxima, umidade relativa do ar e vento foram significativas, no que se refere à explicação dos casos de internações hospitalares por pneumonia. A 
temperatura máxima e umidade relativa do ar estão inversamente relacionadas com as hospitalizações por pneumonia em crianças menores de nove anos no município de Campina Grande (PB), ou seja, enquanto o número de internações por pneumonia cresce, por exemplo, as variáveis em estudo tendem a diminuir. Já as variáveis temperatura média e vento apresentaram uma relação positiva, com a morbidade em estudo.

Considerando a influência das variáveis temperatura do ar, umidade relativa e vento nos registros hospitalares por pneumonia. Torna-se possível avaliar o risco para a saúde da população em razão dos dados climáticos. Podendo ser implementadas medidas para o planejamento e a implementação de ações que facilitem o atendimento e internações por pneumonia na faixa etária em estudo evitando assim complicações da referida morbidade, em períodos nos quais há uma intensidade de registros por pneumonia que são outono e inverno.

\section{REFERÊNCIAS}

ALEIXO, N. C. R.; SANT'ANNA NETO, J. L.. Condicionantes climáticos e internações por pneumonia: estudo de caso em Ribeirão Preto/SP. Revista do Departamento de Geografia USP, v.27, p.1-20, 2014

ALONSO, W. J.; VIBOUD, C.; SIMONSEN, L.; HIRANO, E. W.; DAUFENBACH, L. Z.; MILLER, M. A.. Seasonality of influenza in Brasil: a traveling wave from the Amazon to the subtropics. American Jornal Epidemiology, v.165, n.12, p.1434-42, 2007.

ANDRADE, D. O.; BOTELHO, C.; SILVA JÚNIOR, J. L. R.; FARIAS, S. S; RABAHI, M. F.. Sazonalidade climática e hospitalizações em crianças menores de cinco anos com doença respiratória, Goiânia/Go. Hygeia, v.11, n.20, p.99 105, 2015.

AYOADE, J. O.. Introdução à climatologia para os trópicos. 12 ed. Rio de Janeiro: Bertrand Brasil, 2012.

BOTELHO, C.; CORREIA, A. L.; SILVA, A. M.C .; MACEDO, A. G.; SILVA, C. O. S.. Fatores ambientais e hospitalizações em crianças menores de cinco anos com infecção respiratória aguda. Caderno de Saúde Pública, v.19, n.6, p.1771-1780, 2003.

BRAGA, A. L. F.; ZANOBETTI, A.; SCHWARTZ, J.. The effect of weather on respiratory and cardiovascular deaths in $12 \mathrm{U}$. S. cities. Environmental Health Perspectives, v.110, n.9, p.859863, 2007.

CONTI, J. B.. Clima urbano. In: Clima e meio ambiente. São Paulo: Atual, 2007.

CURRIERO, F. C.; PATZ, J. A. ROSE, J. B.; LELE, S.. The Association Between Extreme Precipitation and Waterborne Disease Outbreaks in the United States, 1948-1994. American Journal of Public Health, v.91, n.8, p.1194-1199, 2001.

DATASUS. Departamento de informática do SUS. Morbimortalidade geral. Brasília: DATASUS, 2018.

FAÇANHA, M. C.. Impacto da vacinação de maiores de 60 anos para influenza sobre as internações e óbitos por doenças respiratórias e circulatórias em Fortaleza/CE/Brasil. Jornal brasileiro de pneumologia, v.31 n.5, p.415-420, 2005.

FALANGAS, M. E.; THEOCHARIS, G.; SPANOS, A.; VLARA, L. A.; ISSARISE, E. A.; PANOS G.; PEPPAS, G.. Effect of meteorological variables on the incidence of respiratory tract infections. Respiratory Medicine, v.102, p.733-737, 2008.

GONZÁLEZ, D. A.; VICTORA, C. G.; GONÇALVES, H.. Efeitos das condições climáticas no trimestre de nascimento sobre asma e pneumonia na infância e na vida adulta em uma coorte no Sul do Brasil. Caderno de Saúde Pública, v.24, n.5, p.1089-102, 2008.

HETZEL, J. L.; SILVA, L. C. C.; SILVA, L. M. C.. Asma Brônquica. In: Doenças Pulmonares. Rio de Janeiro: Guanabara Koogan, 2008.

IBGE. Instituto Brasileiro de Geografia e Estatística. Dados do Censo de 2018. Rio de Janeiro: IBGE, 2018.

LIMA, E. A.; FIRMINO, J. L. N.; GOMES FILHO, M. F.. A relação da precipitação pluviométrica e os casos de dengue nos estados de Alagoas e Paraíba nordeste do Brasil. Revista brasileira de meteorologia, v.23, n.3, p.264-269, 2008.

LIN, S.; LUO, R. J.; LIU, W. X.; HWANG, S.; CHINERV, R. Extreme Hing Temperatures and Hospital Admissions for Respiratory and Cardiovascular Diseases. Epidemiology, v.20, n.5, p.738-746, 2009.

MENDONÇA, F.; PAULA, E. V.. Meningites no estado do Paraná. Revista RA' EGA, n.14, p.127-143, 2008.

LOWEN, A. C.; MUBAREKA, S.; STEEL, J.; PALESE, P.. Influenza virus transmission is dependent on relative humidity and temperature. PLoS Pathogens, v.3, p.1470-6, 2007.

MACEDO, S. E.; MENEZES, A. M.; ALBERNAZ, E.; POST, P.; KNORST, M.. Fatores de risco para internação por doença respiratória aguda em crianças até um ano de idade. Revista de Saúde Pública, v.41, p.351-8, 2007. 
MIRANDA, L. C.; PARENTE, M.; SILVA, C.. A percepção da dor e alterações climatéricas em doentes reumáticos. Acta Reumatológica Portuguesa, v.32, p.351-61, 2007.

MURARA, P. G.; AMORIM, M. C. C. T.. Clima e saúde: variações atmosféricas e óbitos por doenças respiratórias. Revista Brasileira de Climatologia, v.6, p.79-92, 2010.

O'BRODOVICH, H. M.; HADDAD, G. C.. The functional basis of respiratory pathology and disease. In: CHERNICK, V.; BOAT, T. F.; WILMOTT, R. W.; BUSH, A.. Kendig's Disorders of the Respiratory Tract in Children. 6 ed. Philadelphia: W. B. Saunders, 1998.

OMONIJO, A. G.; OGUNTOKE, O.; MATZARAKIS, A.; ADEOFUN, C. O.. A Study of Weather Related Respiratory Diseases in Eco-climatic Zones. African Physical Review, v.5, p.41-56, 2011.

PITTON, S. E. C.; DOMINGOS, A. E.. Tempo e doenças: efeitos dos parâmetros climáticos nas crises hipertensivas nos moradores de Santa Gertrudes/SP. Estudos Geográficos, v.2, n.1, 2004

PONTES, C. C.; LEITE, M. L.; GAVÃO, N.; VIRGENS FILHO, J. S.. Efeitos do clima na saúde: análise das internações de crianças menores de cinco anos por pneumonia no município de Ponta Grossa/PR. Revista Brasileira de Climatologia, Curitiba, v.12, n.18, p.38-52, 2016. DOI: http://dx.doi.org/10.5380/abclima.v18i0.43444
R CORE TEAM. R: A Language and Environment for Statistical Computing. Vienna: the R Foundation for Statistical Computing. 2011.

SANTOS, D. A. S.. Influências das variáveis climáticas na prevalência de Infecção Respiratória Aguda em crianças menores de dois anos no município de Rondonópolis-MT. Rondonópolis. Tese (Doutorado em Recursos Naturais) Universidade Federal de Campina Grande, Campina Grande 2015.

SILVA JÚNIOR, J. L. R.; PADILHA, T. F.; REZENDE, J. E.; RABELO, E. C. A.; FERREIRA, A. C. G.; RABAHI, M.F. Efeitos da sazonalidade climática na ocorrência de sintomas respiratórios em uma cidade de clima tropical. Jornal brasileiro de pneumologia, v.37, n.6, p.759-767, 2011.

SOUZA, A.; FERNANDES, W. A.; PAVÃO, H. G.; ALBREZ, G. L. E. A.. Potenciais impactos da variabilidade climática sobre a morbidade respiratória em crianças, lactentes e adultos. Jornal Brasileiro de Pneumologia, v.38, n.6, p.708-715, 2012.

TIAN, D. D.; JIANG, R.; CHEN, X. J.; YE, Q.. Meteorological factors on the incidence of MP and RSV pneumonia in children. PLoS One, v.12, n.3, p.e0173409, 2017.

VIANELLO, R. L.; ALVES, A. R.. Meteorologia Básica e Aplicações. Viçosa: UFV, 2000.

XU, Z.; HU, W.; TONG, S.. Temperature variability and childhood pneumonia: an ecological study. Enviromental health, v.131, p.2-8, 2014.

A CBPC - Companhia Brasileira de Produção Científica (CNPJ: 11.221.422/0001-03) detém os direitos materiais desta publicação. Os direitos referem-se à publicação do trabalho em qualquer parte do mundo, incluindo os direitos às renovações, expansões e disseminações da contribuição, bem como outros direitos subsidiários. Todos os trabalhos publicados eletronicamente poderão posteriormente ser publicados em coletâneas impressas sob coordenação da Sustenere Publishing, da Companhia Brasileira de Produção Científica e seus parceiros autorizados. Os (as) autores (as) preservam os direitos autorais, mas não têm permissão para a publicação da contribuição em outro meio, impresso ou digital, em português ou em tradução. 